Мусабаева Диана Абаевна

аспирант кафедры экономической социологии и менеджмента

Московского государственного университета

имени М.В. Ломоносова

\section{ОЦЕНКА РЕЗУЛЬТАТИВНОСТИ СОЦИАЛЬНЫХ ПРОЕКТОВ НЕКОММЕРЧЕСКОГО СЕКТОРА: МЕТОДОЛОГИЧЕСКИЕ И ПРАКТИЧЕСКАЯ ПРОБЛЕМЫ}

\begin{abstract}
Аннотация:
В настоящее время наблюдаются тенденции роста интереса некоммерческого сектора к оценке социальных проектов. Тенденция обусловлена тем, что финансирующие организации хотят видеть результат поддерживаемого ими проекта. Однако нельзя не отметить, что данная тенденция позитивно сказывается и на самих НКО, так как повышает их проектную культуру и предоставляет возможность планировать будущую деятельность более качественно. В статье рассматриваются состояние проектной деятельности, сложившееся в некоммерческой сфрере, и практика по оцениванию результативности социальных проектов. Цель исследования заключается в выявлении и анализе методологических и практических проблем оценки результативности социальных проектов, которые отражаются на качестве деятельности некоммерческих организаций. Автор статьи приходит к выводу, что решением выявленных методологических проблем может стать междисциплинарная технология оценки результативности социальных проектов, адаптированная под конкретную цель и конкретные задачи.
\end{abstract}

Ключевые слова:

оценка, социальный проект, результативность, методологические проблемы, некоммерческие организации, социальный результат, социальный эфрект, социальное проектирование.
Musabaeva Diana Abaevna

PhD student Department of Economic Sociology and Management,

Lomonosov Moscow State University

\section{EFFICIENCY ASSESSMENT OF SOCIAL PROJECTS IN THE NON-PROFIT SECTOR: METHODOLOGICAL AND PRACTICAL CHALLENGES}

Summary:

Nowadays, there is a growing interest of the non-profit sector in the assessment of social projects. This trend is due to the fact that funding organizations want to see the result of the project they support. However, it should be noted that the above-mentioned trend has a positive effect on the non-profit organizations as well, as it enhances their project culture and provides an opportunity to plan future activities more efficiently. The research examines the state of the project activity prevailing in the non-profit sphere and the efficiency as sessment of social projects. The purpose of the study is to identify and analyze the methodological and practical issues of evaluating the impact of social projects on the quality of the non-profit organizations activities. The author concludes that the methodological problems can be solved by the interdisciplinary technology for assessing the efficiency of social projects adapted to the specific goal and objectives.

Keywords: assessment, social project, efficiency, methodological challenges, non-profit organizations, social results, social impact, social design.

Социальная сфрера общества представляет собой многогранное, динамичное явление, объединяющее различные отрасли, социальные системы и подсистемы, а также комплекс взаимоотношений, которые возникают между различными социальными группами и слоями населения. Социальная сфера обеспечивает создание условий для духовного и физического развития человека. Степень развития и состояния социальной сферы отражается на показателях качества жизни населения. Мониторинг показателей качества жизни позволяет выявить сильные и слабые стороны жизнедеятельности общества. Именно слабые стороны сигнализируют о присутствии социальных проблем. Существующие социальные проблемы предъявляют высокие требования к социальному поведению государства, бизнеса и некоммерческих организаций. Наиболее эффективным методом в решении социальных проблем, учитывающим специфику различных категорий населения, является разработка социального проекта [1].

Понятие «социальный проект» развивалось в тесной связи с рядом социологических концепций и подходов: социальная утопия [2; 3; 4], социальная инженерия [5; 6], структурно-фрункциональный подход [7], френоменология [8, с. 106], концепция социальной трансформации [9]. В дальнейшем данное понятие, в отличие от понятия «проект», которое разрабатывалось как в экономической науке, так и в инженерии, исследовалось в социологических школах в контексте социального проектирования. 
В отечественной социологической науке сформировались три подхода к социальному проектированию: объектно, проблемно и субъектно-ориентированный. С позиции объектно ориентированного подхода социальный проект рассматривается как создание или усовершенствование (реконструкция) объекта, который выполняет важную социокультурную функцию в обществе. В рамках данного подхода были разработаны концепции Ж.Т. Тощенко [10], Г.А. Антонюка [11], В.И. Курбатова [12] и др. Проблемно ориентированный подход (Т.М. Дридзе [13] и др.) акцентирует внимание на диагностическую, прогнозную составляющую. В рамках данного подхода социальный проект представляет собой научно обоснованное и многовариантное решение социально значимых проблем. Субъектно-ориентированный, или тезаурусный подход, был разработан В.А. Луковым [14]. Данный подход рассматривает тезаурус создателя проекта в качестве основного источника проектной идеи. По мнению В.А. Лукова, социальное проектирование представляет собой конструирование индивидом, группой или организацией действия, направленного на достижение социально значимой цели и локализованного по месту, времени и ресурсам [15, с. 7].

Социальное проектирование представляет собой процесс, связанный с созданием прототипа социальных объектов, качеств, процессов и отношений [16, с. 15].

Несмотря на то что сейчас понятия «проект», «проектная деятельность», «социальное проектирование» широко распространены, доля некоммерческих организаций, осуществляющих свою деятельность в формате проекта, остается незначительной. Об этом фракте свидетельствует активность некоммерческого сектора в конкурсе Фонда президентских грантов, представляющего собой наиболее формализованную площадку для оценки состояния проектной практики [17]. Во втором конкурсе 2018 г. было подано 8560 заявок, в то время как по данным Минюста на июль 2018 г. в России зарегистрировано 219967 некоммерческих организаций, имеющих разные организационно-правовые фрормы [18]. Для участия в конкурсе на получение гранта некоммерческим организациям необходимо разработать и представить социально значимый проект. Участники конкурса, не прошедшие отбор, отмечают, что возникают трудности и проблемы в написании обоснованного социального проекта, который бы соответствовал конкурсным требованиям, вследствие чего они допускают ошибки и не получают так необходимую им грантовую поддержку [19, с. 308].

Обоснованный социальный проект подразумевает не только описание социальной значимости проекта, но и разработку логической цепочки, которая бы объясняла логику проекта: как вкладываемые проектные ресурсы преобразуются в результаты разного уровня. Поэтому в настоящее время отмечается тенденция роста интереса к оценке социальных проектов, к методам оценки социальных результатов со стороны некоммерческих организаций: финансирующие организации хотят видеть результат поддерживаемых ими проектов.

Переходя к более общему контексту, дадим определение понятию «оценка проекта». Так, А.С. Автономов и Н.Л. Хананашвили определяют оценку социальных проектов как вынесение суждения о достоинствах и недостатках проекта с выводом о степени годности его для реализации, о ходе его выполнения, о достигнутом результате, об эффректе после его завершения [20, с. 11].

К одной из главных трудностей оценки результата социального проекта относится «смешанная природа» создаваемых ценностей [21, с. 37]. «Смешанная природа» ценностей заключается в объединении экономических и социальных результатов проекта, что приводит к отсутствию единого стандарта ее оценки. Сегодня используются многочисленные качественные и количественные методы оценки социальных проектов, часть которых акцентирует внимание на социальных результатах, другая - на социально-экономических результатах. Английский ученый С. Грин считает, что при оценке ценности, создаваемой социальным проектом, необходимо учитывать и количественные (финансовые), и качественные результаты [22]. Смешанная природа создаваемых в результате реализации проекта ценностей не позволяет вычленить влияние и участие конкретных мероприятий (например, труд волонтеров) в ее создании.

Оценка достижения социальных результатов проекта опирается на вышеупомянутую логическую цепочку, или модель проекта, которая представляет собой инструментарий, описывающий связи между элементами проекта: ресурсами, действиями, непосредственным результатом, социальным результатом и социальным эффректом. Здесь следует более подробно остановиться на последних трех элементах цепочки и конкретизировать их.

Непосредственный результат (количественный результат) - все, что непосредственно производится в ходе деятельности организации, реализующей социальный проект (количество подготовленных и распространенных информационных материалов, количество лекций и консультаций и др.).

Социальный результат - результат, отражающий позитивные изменения в ситуации с целевой аудиторией проекта (изменения в отношении к чему-либо, повышение информированности и др.).

Социальный эфффект - то, что изменилось в структуре проблемы в результате деятельности по проекту. 
Непосредственные и социальные результаты имеют принципиальное отличие. Данное отличие заключается в том, что социальные результаты - это не то, что «сделал» сам проект, а последствия проектной деятельности для целевой аудитории. Непосредственные результаты проекта не дают ответа на вопросы, получены ли запланированные результаты и достигнута ли цель проекта, в то время как социальные результаты представляют собой результаты на уровне цели проекта.

В работе «Мониторинг результативности в общественном секторе» Гарри П. Хатри обозначил, что в отчетной практике часто происходит смешение непосредственного результата и социального результата, т. е. деятельности и достижения [23]. Данное положение распространяется и на российскую практику. Так, социолог, эксперт-аналитик некоммерческой организации «Эволюция и филантропия» Н. Фреик, пообщавшись с представителями российских НКО, описала, как они оценивают эффрективность своей деятельности. Среди основных критериев эффективности некоммерческие организации выделяют и непосредственные результаты: количество обращений в НКО, количество проведенных мероприятий, оказанных услуг, количество предпринятых действий - установленных ящиков для пожертвования, проведенных фандрайзинговых акций и пр.

В методологическом плане наибольшую сложность представляет оценка социальных эффректов проектной деятельности, или долгосрочного воздействия проекта, которое отражает изменения в структуре проблемы на уровне общества. Трудность оценки социального эффекта заключается в том, что достаточно проблематично доказать, что именно деятельность по данному социальному проекту привела к позитивным изменениям в социальной проблеме, отраженным в статистической разнице по отношению к первоначальному состоянию. Для того, чтобы использовать статистические методы оценки социального эффекта от реализованного проекта, необходимо создать «вакуумные условия», что в реальности неосуществимо. Поэтому даже позитивные изменения в значениях показателей по социальной проблеме не являются гарантией того, что данные позитивные изменения есть результат реализованного проекта, а не действия других внешних или внутренних факторов.

Другая методологическая проблема связана с самой оценочной базой, которая включает в себя такие необходимые компоненты, как четко прописанные цели и результаты проекта, по которым оценивается, достигнута ли цель проекта. Четко прописанные цель и результаты проекта предполагают наличие индикаторов, которые будут измерять результаты проекта и значения индикаторов, при которых цель будет считаться достигнутой. И именно выбор системы индикаторов, связывающих цель социального проекта и результаты, а также значения индикаторов являются наиболее сложным этапом проектного планирования [24, с. 46]. Здесь стоит также отметить, что при выборе системы индикаторов и оценке результатов социального проекта присутствует субъективность, поскольку в данной области нет стандартов оценивания, что влияет на объективность осуществления данной процедуры.

Таким образом, оценка результативности социальных проектов некоммерческого сектора характеризуется рядом проблем: одни из них являются специфичными, выраженными в низкой проектной культуре, другие - общими, касающимися процедуры оценки результативности любых социальных проектов. Общие проблемы относятся к методологии оценивания и кроются в самой оценочной базе. Вопрос о технологии оценки результативности социальных проектов, нивелирующей методологические проблемы, остается открытым. В практической реализации можно говорить о более или менее эффективном комбинировании методов оценки социальных проектов под конкретные задачи. Задачи социального проекта нередко носят междисциплинарный характер, поэтому привлечение специалистов из соответствующих предметных областей для проверки «теории» проекта и оценивания его результатов позволит повысить качество оценки.

\section{Ссылки:}

1. Автономов А.С., Хананашвили Н.Л. Оценка социальных проектов / под общ. ред. А.С. Автономова. М., 2014. 240 с.

2. Платон. Государство / пер. А.Н. Егунова // Платон. Собрание сочинений : в 3 т. Т. 3, ч. 1. М., 1971

3. Мор Т. Утопия / пер. с лат. Ю.М. Каган. М., 1978.

4. Тоффлер Э. Шок будущего : пер. с англ. М., 2002

5. Поппер К. Открытое общество и его враги : пер. с англ. Т. 1. М., 1992.

6. Гастев А.К. Как надо работать. Практическое введение в науку об организации труда. М., 1966.

7. Парсонс Т. О социальных системах. М., 2002. С. 721-732.

8. Шюц А. Избранное: Мир, светящийся смыслом. М., 2004.

9. Заславская Т.И. Современное российское общество: социальный механизм трансформации. М., 2004.

10. Тощенко Ж.Т. Социология. М., 1994

11. Антонюк Г.А. Социальное проектирование и управление общественным развитием: теоретико-методический аспект. Минск, 1986.

12. Курбатов В.И., Курбатова О.В. Социальное проектирование. Ростов н/Д., 2001. 
13. Прогнозное социальное проектирование: теоретико-методологические и методические проблемы / отв. ред. Т.М. Дридзе. М., 1994.

14. Луков В.А. Социальное проектирование. М., 2007

15. Там же. С. 7

16. Ангела О.Ю. Методы социального прогнозирования и проектирования. Ростов н/Д., 2018.

17. Офрициальный сайт Фонда президентских грантов [Электронный ресурc]. URL: https:/президентскиегранты.pф/ (дата обращения: 20.07.2018).

18. Информационный портал Министерства юстиции Российский Федерации [Электронный ресурс]. URL: http://unro.minjust.ru/NKOs.aspx (дата обращения: 20.07.2018).

19. Вишнева К.В., Прохоренко Ю.И. Проблемы реализации и перспективы развития проектной культуры и деятельности социально ориентированных некоммерческих организаций // Ученые заметки ТОГУ. 2017. Т. 8, № 1. С. 306-309.

20. Автономов А.С., Хананашвили Н.Л. Оценка социальных проектов / под общ. ред. А.С. Автономова. 2-е изд., стер. М., 2015. 236 c

21. Оценка экономической и социальной эффективности добровольческой деятельности / под ред. И.В. Мерсияновой. М. ; СПб., 2018.

22. Green S. Creating a Tool for Measuring the Social Value of Design // The Design Journal. 2017. Vol. 20. P. 662-672. https://doi.org/10.1080/14606925.2017.1352689.

23. Хатри Г.П. Мониторинг результативности в общественном секторе : пер. с англ. М., 2005

24. Табачникова М.Б. Теория и практика управления социальными проектами: организационный, институциональный, территориальный аспект : монография. Воронеж, 2016.

\section{References:}

Angela, OYu 2018, Methods of social forecasting and design, Rostov-on-Don, (in Russian).

Antonyuk, GA 1986, Social design and social development management: the theoretical and methodological aspect, Minsk, (in Russian).

Avtonomov, AS (ed.) \& Khananashvili, NL 2014, Evaluation of social projects, Moscow, 240 p., (in Russian)

Avtonomov, AS (ed.) \& Khananashvili, NL 2015, Evaluation of social projects, $2^{\text {nd }}$ ed., Moscow, 236 p., (in Russian). sian).

Dridze, TM (ed.) 1994, Forecasting social design: theoretical and methodological and methodical issues, Moscow, (in Rus-

Gastev, AK 1966, How to work. Practical introduction to the science of labor organization, Moscow, (in Russian).

Green, S 2017, 'Creating a Tool for Measuring the Social Value of Design', The Design Journal, vol. 20 , pp. 662-672. https://doi.org/10.1080/14606925.2017.1352689.

Hatry, HP 2005, Performance measurement getting results, Moscow, (in Russian).

Information portal of the Ministry of Justice of the Russian Federation 2018, viewed 20 July 2018 ,

$<$ http://unro.minjust.ru/NKOs.aspx>, (in Russian).

Kurbatov, VI \& Kurbatova, OV 2001, Social design, Rostov-on-Don, (in Russian).

Lukov, VA 2007, Social design, Moscow, (in Russian).

Mersiyanova, IV (ed.) 2018, Estimation of economic and social efficiency of volunteer activity, Moscow, St. Petersburg, (in Russian)

More, T 1978, Utopia, Moscow, (in Russian).

Official website of the Presidential Grants Foundation 2018, viewed 20 July 2018, <https://президентскиегранты.рф/>, (in Russian).

Parsons, T 2002, The social system, Moscow, pp. 721-732, (in Russian).

Plato 1971, The republic, collected works, in 3 vols., vol. 3, iss. 1, Moscow, (in Russian).

Popper, K 1992, Open society and its enemies, vol. 1, Moscow, (in Russian).

Schütz, A 2004, Selected works: world full of meaning, Moscow, (in Russian).

Tabachnikova, MB 2016, Theory and practice of social projects management: arrangement, institutional, territorial aspect:

monograph, Voronezh, (in Russian).

Toffler, A 2002, Future shock, Moscow, (in Russian).

Toshchenko, ZhT 1994, Sociology, Moscow, (in Russian).

Vishneva, KV \& Prokhorenko, Yul 2017, 'Implementation problems and development prospects of the project culture and the activities of community-focused non-profit organizations', Uchenyye zametki TOGU, vol. 8, no. 1, pp. 306-309, (in Russian).

Zaslavskaya, TI 2004, Modern Russian society: the social mechanism of transformation, Moscow, (in Russian). 